\title{
HACIA UNA LECTURA DE "LA PALABRA DICHA" de Octavio Paz
}

La obra de Octavio Paz se ha caracterizado desde sus inicios por la conjunción del poeta con el ensayista. Este paralelismo inicial se ha ido cumpliendo en las diferentes etapas de una vasta obra que tiene ya cuarenta años de vida.

Uno de los temas constantes en la obra ensayística de Paz es la indagación sobre el quehacer poético; por ende, en torno al lenguaje y la experiencia poética misma. La continua y fecunda reflexión sobre esta problemática ha cimentado una teoría poética expresada orgánicamente, sobre todo, en El arco y la lira (1956). Sin embargo en este ensayo se refleja la experiencia de la escritura de la poesía del periodo que va de 1935 a 1957, reunida en Liberlad bajo palabra (1957).

La correlación entre teoría poética y poesía tiene, posteriormente, otro contacto especialmente importante. En 1957 la segunda edición de El arco y la lira apareció con un nuevo epílogo, "Los signos en rotación", el cual aporta a la teoría poética reiteraciones y respuestas nuevas a las preguntas originales que dieron vida al libro. ${ }^{1} \mathrm{El}$ epílogo, calificado por el mismo $\mathrm{Paz}$ como "[...] suerte de manifiesto poético"," se refiere, sobre todo, al espacio literario, ${ }^{3}$ cuya concepción parte fundamentalmente de la experiencia que suscitó en Paz la lectura del poema "Un coup de dés", de Mallarmé; pero también refleja la experiencia propia de la escritura de poemas reunidos en Salamandra (1958-1961) y Ladera este (1962-1968). Las convergencias de esta etapa lle-

1 Posteriormente, Paz ha profundizado en algunas de las respuestas expresadas en El arco y la lira, en Los hijos del limo. Del romanticismo a la vanguardia, Seix Barral, Barcelona, 1974.

Ibid.; p. 11 .

3 Yaz concibe el espacio literario como un elemento dinámico del poema; en este sentido coincide con Maurice Blanchot. Cf: L'espace littéraire, Gallimard, Paris, 1965. 
gan a su apogeo, quizás, en "Blanco", extenso poema escrito en 1966.

El enfrentamiento de la teôría y la creación poética de Paz constituye, ciertamente, una vasta tarea. En el presente estudio pretendemos ejemplificar una parte mínima del universo de relaciones. Para tal efecto, hemos elegido un poema de Salamandra, "La palabra dicha".

Nuestro estudio se fundamentará en algunos de los principios constitutivos de la poética jakobsoniana, ${ }^{4}$ los cuales permiten descubrir las relaciones que el poema, como objeto lingüístico, instaura en los niveles fónico, morfosintáctico y semántico que lo integran, y que evidencian un fenómeno que Jakobson ha descrito así:

En la poesía se hacen evidentes los elementos constructivos de todos los niveles lingüísticos desde la red de sus rasgos distintivos hasta la disposición del texto entero. La relación en tre el significante y lo significado, válida para todos los niveles lingüísticos adquiere un valor particular en el verso, donde el carácter introvertido de la función poética llega a su apogeo. Se trata, para decirlo en palabras de Baudelaire, de 'una compleja e indivisible totalidad' en la que todo es significativo, recíproco, convertible, correspondiente, y en la que una mezcla perpetua de sonido y sentido establece, entre estos dos elementos, una relación a veces paronomástica y anagramática, a veces figurativa ( $y$ en ocasiones onomatopéyica). 5

$\mathrm{El}$ análisis del poema presupone las características del discurso poético postulado por Jakobson, de la proyección del "[... principio de la equivalencia del eje de selección al eje de combinación", $n^{\text {in }}$ que permite reunir los haces de equivaIencias entramados en la materia verbal.

He aquí el texto del poema:

- Cf. particularmente "Lingüística y poética", en Ensayos de lingüistica general, Seix Barral, Barcelona, 1975; pp. 345-395.

s "P.S.", Plural, vol. II, n? 3, 1973, p. 6. Plural publico una parte de este trabajo de Jakobson. El artículo integro figura en Questions de poítique, Du Seuil, Paris, 1973; pp. 485-504.

" "Lingüística y poética", en op. cit.; p. 360. 


\section{LA PALABRA DICHA}

I 1 La palabra se Ievanta

2 De la página escrita,

3 La palabra,

4. Labrada estalactita,

5 Grabada columna

6 Una a una letra a letra.

7 El eco se congela

8 En la página pétrea.

II 9 Ánima.

10 Blanca como la página,

11 Se levanta la palabra.

12 Anda

13 Sobre un hilo tendido

14 Del silencio al grito,

I5 Sobre el filo

16 Del decir estricto.

17 El oído: nido

18 O laberinto del sonido.

III 19 Lo que dice no dice

20 Lo que dice: ¿cómo se dice

21 Lo que no dice?

$22 . \quad$ Dí

23 Tal vez es bestial la vestal.

IV 24 Un grito

25 En un cráter extinto:

26 En otra galaxia.

27 ¿Cómo se dice ataraxia?

28 Lo que se dice se dice

$29 \mathrm{Al}$ derecho y al revés.

30 Lamenta la mente

31 De menta demente:

32 Cementerio es sementero,

33 Simiente no miente.

V 34 Laberinto del oído, 35 Lo que dices se desdice 
36 Del silencio al grito

37 Desoído.

\section{38 Inocencia y no ciencia: \\ 39 Para hablar aprende at callar.}

Advertimos que la segmentación del poema responderá a la disposición sintáctica de los versos, es decir, que los segmentos serán las oraciones gramaticales.

La estrofa 1 contiene tres oraciones yuxtapuestas. Los versos 1 ' y 2 forman la oración $a^{7}$ - "La palabra se levanta de la página escrita"-; los siguientes cuatro $(3,4,5$ y 6$)$ la oración $b$ - "La palabra, labrada estalactita, grabada columna una a una letra a letra"-, y el último par de versos (7 y 8) la oración $c-$-El eco se congela en la página pétrea".

La estructura de las oraciones $a$ y $c$, localizadas en los versos iniciales y finales de la estrofa, acusan un paralelismo en la distribución de sus componentes. Ambas presentan el sujeto, más el verbo reflexivo antecedido del pronombre personal "se", objeto directo, eqụivalente semántico del sujeto, y seguido de un complemento circunstancial. En cambio la estructura de la oración $b$, integrada por los versos centrales de la estrofa, resulta desemejante frente a la $a$ y la $c$ porque en la predicación del sujeto se elide el núcleo verbal. En efecto, el verso 6 Una a una letra a letra parece ser complemento de un "se levanta". tácito.

Por otra parte notamos que la similitud sintáctica de las oraciones $a$ y $c$, "La palabra se levanta de la página escrita" y "El eco se congela en la página pétrea", comporta relacionẹs de afinidad y oposición en el nivel morfológico. El sujeto de la oración $a$, "La palabra", es de género femenino, en tanto que el de la oración $c$, "el eco" es masculino, pero ambos son sustantivos inanimados.

La oración $b$, "la palabra, labrada estalactita, grabada columna, una a una, letra a letra" reitera el sujeto de la oración $a$, "la palabra", y lo amplía con las aposiciones "labrada

- Marcamos con letras las oraciones y se entrecomillan cuando se citan integra o parcialmente. Subrayamos la cita integra de versos y encerramos con una comilla los semas. 
estalactita" y "grabada columna". La elisión que suponemos de la forma verbal "se levanta", convierte a la oración en una enumeración de sustantivos y adjetivos, todos de género femenino, cuya similitud morfológica es reiterada por las equivalencias fónicas producidas por aliteraciones (la-la-la-, bra-bra) .

La descripción de las relaciones fundadas en la contigüidad de los versos de la estrofa 1 nos permiten aproximarnos a las equivalencias semánticas creadas tanto en las relaciones sintagmáticas u horizontales como en las paradigmáticas o verticales, que evidencian el modo peculiar de proyección de semas.

En principio la forma verbal "se levanta" (1) privilegia el sema nuclear 'movimiento' con respecto al sujeto, "la palabra". Después se despliega hacia los versos siguientes creando equivalencias mediante los semas 'erguimiento' y 'verticalidad' (en tanto que la forma verbal reflexiva implica la acción de poner en posición vertical) con "estalactita" y "columna". El tejido de equivalencias semánticas se amplía horizontalmente con la modificación ejercida por los adjetivos "labrada" y "grabada", los cuales descubren una semejanza, en una lectura rertical, con "página escrita", posible gracias al sema de 'labor' que comportan ambos adjetivos. Dicha 'labor' evoca tanto la artística - la del poeta- como la artesanal - tipográfica--, que implica la página escrita y que la convierte en un conjunto de columnas grabadas.

La reiteración, lograda por la ausencia, de la forma verbal "se levanta", elidida en la oración $b$, despliega otros semas además del nuclear 'verticalidad'. Dichos semas provienen de otra palabra, "construir", que nos permite interpretar cabalmente el significado de "levantar". Contextualmente, "construir" sería equivalente de "levantar", por lo cual el sema 'construcción' se convierte en interpretante sémico ${ }^{8}$ de "se levanta". 'Construcción' recae en los versos que le anteceden y le suceden, es decir, en "Iabrada estalactita" $y$

${ }^{8}$ Nos basamos en el hecho que para ser interpretado un signo requiere de otro signo o conjunto de signos. Este último signo, es denominado por Charles Sanders Peirce, interpretante del primer signo. Cf. La ciencia de la semiótica, Nueva Visión, Buenos Aires, 1974; p. 22. 
"grabada columma" y. "una a una, letra a letra". Por otra parte este último verso $(6)$, abarca una serie de equivalencias por semejanza, en línea verticaly con componentes de versos que le anteceden "El pronombre "una" posee el sema 'aislamiento', el cual 'lo convierte en metonimia de "labrada estalactita" (4), "grabada columna" (3) y "página escrita" (2). Simultáneamente todo el verso 6, Una a una letra a letra puede ser interpretado mediante el sema 'sucesión', el cual refuerza la semejanza, en tanto que la suma de los componentes del sintagma es idéntica a la disposición de las palabras en la página escrita.

La consecuencia más importante del análisis sémico de los versos centrales de la estrofa 1 es que la elisión del verbo "se levanta" no impide la presencia de todos sus semas contextuales.

El último par de versos de la estrofa 1, El eco se congela. En la página pétrea, sintácticamente semejante al primer par, y cuyo sujeto es una sinécdoque de aquél, en tanto que "eco" es la resonancia de "la palabra", conforma en la predicación de antítesis ${ }^{9}$ con el primer par de versos (oración b) , integrada por "se congela" y "se levanta", perceptible en los semas 'estaticidad' del primero y 'movimiento' del segundo. Sin embargo la antítesis va seguida de la equivalencia en los complementos circunstanciales de ambas oraciones, "página escrita" y "página pétrea", resultado de la interpretación de los semas 'dureza? y 'rigidez' de "se congela", que comparten tanto con "columna" como con "estalactita".

Integrando el análisis sémico de la estrofa I del poema, es posible observar que los semas nucleares privilegiados se distribuyen de la siguiente manera: 'erguimiento' y 'verticalidad' aparecen en los versos 1, 3, 4 y 5; 'estaticidad' en los versos 2, 5, 7 y 8, mientras que 'movimiento' sólo en dos versos, 1 y 6; y el sema contextual 'construcción' en los versos $2,4,5,6$ y 8 . Dentro de tal distribución tenemos que é

- "La antitesis [...] es una figura que se da por repetición en el sentido, en la cual se enuncia $A$ y se añade: $A$ no es no $A^{\prime \prime}$. Cf. J. Dubois, et $a l$, Rhétorique général, Larousse, Paris, 1970; pp. 195 y 136. 
sema nuclear 'verticalidad' se une con 'horizontalidad' en los versos 2,6 y 8 .

La disposición de las ocurrencias sémicas de la eștrofa I permite notar que la equivalencia sintáctica afin de los versos extremos de la estrofa ( 1 y 2, y 7 y 8 ) comporta semánticamente la antítesis entre 'movimiento' y 'estaticidad', expresada en La palabra se levanta / El eco se congela, pero que entre dichos elementos se da la combinación sémica 'verticalidad' — 'horizontalidad'.

Precisamente entre la pareja antitética las aposiciones "labrada estalactica" (b, 4) y "grabada columna" (b, 5) comportan una precisión fundamental dentro del tejido de la significación. El título del poema "La palabra dicha", expresado en 1 y 2 como La palabra se levanta/De la página escrita, nos indica el justo momento de la lectura del poema, el instante en que la palabra es pronunciada por un lector, acto qu ela yergue de la página y la pone en movimiento. Pero antes de que ocurra el movimiento, la palabra es "labrada estalactita", es decir, que pende de modo análogo a las estalactitas, de arriba hacia abajo, lo cual permite inferir la presencia de un poeta que las ha depositado en la página. Tal inferencia se apoya, por lo demás, en otro poema de Octavio Paz, "La palabra escrita", 10 el cual resulta imprescindible para la cabal interpretación del poema objeto de análisis. Sobre el acto de escribir expresa el poema: "Ya escrita la primera/Palabra (cuatro, cinco)/No acaba de caer la piedrecilla, Mira tu cara mientras cae, cuenta/La cuenta vertical de la caída)/." En efecto, la "cuenta vertical de la caida" suponemos es el acto en que el poeta convierte a la palabra en "labrada estalactita". La caída vertical origina, posteriormente, la conversión de las palabras en "grabada columna" mediante la labor tipográfica de disponerla en la página.

Con la reiteración del movimiento de la palabra enfática con su contrario, la elisión de la forma verbal "se levanta", en el verso 5 se presenta, como ya señalamos, la combina-

10 Este poema antecede a "La palabra dicha" en Salamandra, Mortiz, México, 1977; pp. 29-30. 
ción de los semas 'verticalidad' - 'horizontalidad' en el 6 , Una a una letra a letra, que expresa el movimiento de cada palabra dichaty de cada uno de sus compönentes, las grafías. Este hecho remite tanto a la disposición de todas ellas en el sintagma, esto es, a la horizontalidad en la que se ubican en la página, así como a la verticalidad que ocurre cuando se yerguen en el momento en que se desprenden de la página, pero también, finalmente, a la conjunción de horizontalidad y verticalidad que muestran las palabras en la disposición que ellas toman en la página, efecto este último perceptible visualmente.

La estrofa II del poema está integrada, igual que la $I$, por tres oraciones. La primera de ellas, $d$, "Ánima, blanca como la página se levanta la palabra" $(9,10$ y 11) presenta una aposición metafórica en el sujeto "la palabra", compuesta por dos miembros, "ánima" y "blanca como la página", hecho que, por un lado, se asemeja a la oración $b$ de $I$, en la cual hay una aposición compuesta por dos miembros. $Y$ por otro lado reitera tal disposición bimembre en un complemento, ocurrida en la oración $e$, de II (12 a 16), pero en etse caso en el predicado, donde tenemos los complementos circunstanciales "sobre un hilo tendido del silencio al grito" y "sobre el filo del decir estricto".

La oración $f$ de II, el último par de versos, El oido:nido/ $O$ laberinto del sonido, muestra desemejanza sintáctica con las dos oraciones que la preceden, puesto que carece de núcleo verbal, elisión marcada con los dos puntos que sustituyen a "es". Sin embargo la oración $f$ comparte con las oraciones que le anteceden la estructura bimembre del predicado, cuyas partes están separadas por una disyunción. Notamos, además, que en esta oración ocurre lo mismo que en la $b$, de I, es decir, que la elisión de la forma verbal "es" la convierte en una sucesión de nombres, en la cual se fundan equivalencias fónicas manifestadas por las rimas entre "oído" y "nido" y entre "nido" y "sonido". Añadamos que los nombres son todos de género masculino, rasgos morfológicos presentes también en la oración anterior, $e$, compuesta por sústantivos y adjetivos masculinos, rasgo opuesto a los nombres de la estrofa I, de género femenino. Las afinidades y las opo- 
siciones de los géneros de los nombres están perfectamente delimitadas en las estrofas I y II: los femeninos corresponden a "palabra" y los masculinos a "eco", la resonancia de ella.

El descubrimiento de las relaciones semánticas instauradas en la estrofa II puede iniciarse con la reiteración de algunos de los semas nucleares del verbo "levanta", de 11 . Advertimos que los semas 'movimiento' y 'erguimiento' muestran una relación semejante a la de los versos 1 y 2 de I, lo cual integra un paralelismo semántico entre los versos iniciales de ambas estrofas ( 1 y 2,910 y 11 ). Por su parte el sema contextual 'construcción' de "levanta" (11) se relaciona retrospectivamente con "ánima" (9) mediante el sema 'energía' que comporta aquélla, así como el de 'principio vital'. A su vez "ánima", como equivalente de "alma", aporta los semas nucleares 'esencial' y 'parte principal', de lo cual podemos inferir que "ánima", aposición de la palabra, es la parte esencial o principal de ella, sería justamente el "significado". La segunda modificación de "la palabra", expresada en el verso 10, "Blanca como la página", reúne dos equivalencias. En primer lugar "blanca" se relaciona verticalmente con "ánima" por cuanto comparten el sema "pureza' y, en segundo lugar, en la contigüidad, el sema 'blancura' crea la equivalencia con "página".

La identidad del matiz aspectual de los verbos "levanta" y "anda" (11 y 12), la iteratividad, permite reiterar las etapas del movimiento de la palabra. "Anda" especifica en los versos centrales de la estrofa (12 a 16) las etapas. Las relaciones fundadas en la contigüidad ponen de relieve un tejido semántico complejo. Notamos que "hilo" (13) mantiene relaciones retrospectivas con "ánima" (9), localizadas en la conjunción del sema contextual 'significado' de éste, y el sema nuclear 'continuación o serie del discurso' connotado por "hilo".

En las relaciones creadas en el sintagma "un hilo tendido del silencio al grito" ocurre la antitesis "silencio"/"grito", cuyos términos equivalen a "callar"/"hablar". La pareja antitética supone un intermedio, en este caso, precisamente el recorrido de la palabra que va desde un silencio hasta un 
grito. El silencio adquiere una fundâmental significación en el poema, sobre todo si conisideramos que su título es "La palabra dicha", y âludé a la lè̀ctura del poema, al acto de decirlo y no de callarlo. A este respecto el propio Octavio Paz precisa en su teoría poética: "La palabra se apoya en un silencio anterior al habla - in presentimiento del lenguaje. El silencio, después de la palabra, reposa en un lenguajees un silencio cifrado. El poema es el tránsito entre uno y otro silencio." 11 El postulado de Paz ubica con exactitud tanto el acto de escribir el poema como el de leerlo.

El paralelismo sintáctico de los versos 15 y 16 con respecto a los que le anteceden, 1.3 y 14 , comporta, en principio, la homofonía "filo" $\mid$ "hilo". Pero las relaciones semánticas de "El filo del decir estricto" añaden los semas nucleares "borde' y 'orilla' de "filo", los cuales confluyen hacia "decir estrcito", adjetivo este último que posee los semas nucleares 'estrechez' y 'ajustabilidad'; con ellas se producen simetrías que aportan la idea de que la "palabra"-_'significado' (Ani$m a$ ) toca sólo el borde del decir justo, pero que a la vez lo recorre, con lo cual se asemeja, verticalmente, con Anda/ Sobre un hilo tendido (12 y 13 ).

Que el 'significado' de "la palabra" ande por la orilla del "decir estricto" descubre el hecho fundamental de que no se ajusta a una sola designación sino que, por el contrario, conserva su polisemia original. Tal es la condición que adquiere la palabra en su movimiento; tal sería, además, la consecuencia de la lectura del poema; de aquí su acentuada densidad.

Advertimos, por otra parte, que las expresiones "anda sobre un hilo tendido" y "anda sobre el filo", evocan las frases coloquiales "andar en la cuerda floja" y "estar en el filo de la navaja", que connotan riesgo y peligrosidad. Otra expresión equivalente sería "pender de un hilo" que implica, además de riesgo, poca estabilidad. Este sema nos conduce a 'precariedad', interpretante que reúne la significación de los versos 12 a 16 , homóloga a la concepción del silencio expresada por Octavio Paz y citada anteriormente. Lo precario y

"Corriente alterna, Siglo XXI, México, 1967; p. 74. 
peligroso reside en el proceso que se desencadena en el acto de leer el poema, proceso singular que será descrito más adelante.

En el último par de versos de la estrofa II, 17 y 18 , observamos primeramente que uno de los semas nucleares de "oído", 'percepción de sonidos', se relaciona verticalmente con "silencio" y "grito". En segundo lugar ocurre una sinécdoque entre "laberinto" y "oído". A su vez "laberinto" aporta el sema nuclear 'confusión' y especifica la última etapa del movimiento y recorrido de la "palabra dicha", señalando que el 'significado' ("ánima") recorre el discurso ("hilo" y "filo") y se confunde finalmente en el "laberinto del sonido". Esta interpretación nos permite afirmar que en la estrofa II se reitera el movimiento de la palabra, pero que, además, tras el erguimiento de ella, y por ende del 'significado', ocurre su tránsito, en el cual las resonancias sufren confusiones. Precisamente estas equivalencias nos remiten a "el eco", verso 7, I, para confirmarnos que éste sería el otro componente de la palabra, el 'significante'.

Si revisamos la disposición sintáctica de los versos 17 y 18 advertimos que la disyunción abarca el hecho de que el "oído" sea tanto "nido" como también "laberinto del sonido". Ocurre entonces una confluencia de los semas nucleares 'percepción de sonidos' de "oído", y "lugar de procreación' y 'principio o fundamento', de "nido". Sin embargo cuando el "oído" es "laberinto", es decir, cuando interviene el sema 'confusión', se convierte en opuesto de "nido" porque lo que origina es la "confusión del sonido". En estos versos se da la confluencia de una cuasisinonimia y de una antítesis entre "oído" y "nido".

La estrofa III del poema presenta un complicado juego sintáctico que ofrece la posibilidad de varias lecturas. Describiremos la que se ajusta a la segmentación por oracioneses, como sigue: oración $g$, "lo que dice no dice lo que dice", abarca el verso 19 y parte del 20. Oración $h$, "¿Cómo se dice lo que no dice?", 20 (segunda parte) y 21. Oración $i$, "Di", 22. Y oración $j$, "Tal vez es bestial la vestal", 23.

La oración $g$, "Lo que dice no dice lo que dice", posee como sujeto la frase de sustantivo "Io que dice" y como pre- 
dicado la misma frase. La oración impersonal $h$, "CCómo se dice lo que no dice?", presenta en el sujeto el representante de una persona indefinida, pero reitera el objeto directo de la oración $g$, "lo que". La oración $i$, "Di", posee sujeto tácito (tú), y la oración $j$, última de la estrofa es predicativa y contiene la modificación adverbial "tal vez".

La disposición sintáctica de los versos de la estrofa III evidencia que las primeras tres oraciones $\langle g, h, i\rangle$, tienen como sujeto y como predicado frases con formas del verbo "decir", negado en la oración $g$, afirmando en la $h$, y enfatizado afirmativamente con el imperativo en la oración $i$. Por otra parte el sujeto de las dos primeras oraciones es indeterminado ("lo que" y "se"), y en la siguiente, tácito. A lo anterior se agrega el predominio en la estrofa de las formas verbales de "decir" frente a sólo dos adjetivos de género femenino, hecho por el cual se crea una desemejanza con las dos estrofas anteriores, en las cuales, por el contrario, predominan los nombres, sustantivos y adjetivos; sin embargo observamos que la función de las oraciones de la estrofa III es sustantiva.

La reiteración del verbo "decir" explicita la antítesis "decir"/"no decir" en las oraciones g y $h$. Semánticamente, y considerando una relación vertical, tenemos que en la oración $g, 19$ y 20 Lo que dice no dice/Lo que dice, se origina una correlación antitética porque el segundo verso desmiente al primero, hecho que se repite de modo idéntico en la oración $h, 20$ y 21 iCómo se dice/Ĺo que no dice? Si de aquí continuamos con las relaciones fundadas en el siguiente verso, 22, $D i$, se añadiría otro término que también desmiente semánticamente al anterior, "Lo que dice". Finalmente, el verso 23, Tal vez es bestial la vestal, introduce la equivalencia entre la dubitación, con el modificador "tal vez", que integra el juego que hay entre afirmación, negación y dubitación.

La correlación antitética de los versos 19, 20 y 21, se resuelve con el enfático "dí" de 22 , hecho que señala la relatividad de la pareja de contrarios. En el poema "La palabra escrita" se alude a este incesante juego con el que se encuentran las palabras del poema: Ya escrita la primera/Palabra 
(nunca la pensada/Sino la otra - ésta/Que no la dice, que la contradice/Que sin decirla está diciéndola).

Las equivalencias fonosemánticas del último verso de la estrofa III (23), Tal vez es bestial la vestal se enfrentan de modo singular a las correlaciones antitéticas de los versos anteriores. En primera instancia el verso presenta aliteraciones (tal, tial, tal, ves, es bes-), pero es importante notar que las repeticiones silábicas idénticas que se dan por pares: -tal -tal y vez-bes- ocurren justamente en la primera y última palabra del verso, y que la fusión de ellas, mediante la inversión del orden habitual de la lectura, producen la misma palabra. En efecto, si leemos de derecha a izquierda "vestal" es igual a "tal vez". La función de estas palabras extremas del verso es, en consecuencia, la de indicar la posibilidad de dos lecturas, es decir, la de trastocar el orden lineal acostumbrado. Pero todavía más importante es el hecho de que en cualquiera de las dos lecturas la nueva palabra ("tal vez" - "vestal") surgida de la inversión silábica, se enfrenta a una palabra semánticamente opuesta, en este caso, "bestial". La antítesis "bestial" /"vestal", perceptible en los semas nucleares de 'animalidad' de la primera y 'divinidad' de la segunda, añadimos, se sustenta en una homofonía.

La significación de la estrofa III permite inferir equivalencias con las relaciones semánticas de la estrofa anterior. En principio la correlación antitética "decir"/"no-decir" equivale a "silencio" y "grito" del verso 14, de manera que tendríamos las parejas "hablar"/"callar" equivalente a "decir"/"no decir". Por otra parte las equivalencias fonosemánticas del último verso de la estrofa III, nos remiten a la resonancia de "la palabra dicha"-- "eco", 1, 7 y a los versos 17 y 18 , II. Con respecto a estos últmos el juego de homofonías del verso 23 es precisamente una ilustración, en dos sentidos. En primer término la pura conjunción de los significantes "bestial" /"vestal" puede confundir en el "laberinto del sonido" la antítesis de los significados. Pero, en segundo lugar, el "oído", que es "nido" (17) puede fundar una nueva palabra. Una y otra capacidad del "oído" —a su vez antitética, como ya se señaló - alude a la permanente fun- 
dación de antinomias que ocurre en el encuentro de las palabras.

La estrofa IV del poema constituye una reiteración sintáctica de algunas de las relaciones advertidas en la estrofa III. La primera oración, $k$ (24, 25, 26 y 27); "Un grito en un cráter extinto: en otra galaxia, ¿cómo se dice ataraxia?", está formada por una oración interrogativa situada al final, y antecedida por dos complementos que parecen pertenecer a un verbo elidido. En efecto, la respuesta a la interrogación "¿Cómo se dice ataraxia?" suponemos que sería "En otra galaxia, se dice un grito en un cráter extinto". La oración siguiente, $l$, "Lo que se dice se dice al derecho y al revés" resulta semejante a las oraciones $g$ y $h$, de III. Tenemos que el sujeto es una oración impersonal y el predicado incluye una parte de los componentes del sujeto, el pronombre cuasirreflejo "se", aunque esta oración posee el complemento circunstancial "al derecho y al revés", el cual también se presenta en la siguiente oración, $m$, "Lamenta la mente de menta de mente". Mientras que la oración $n$, "Cementerio es sementero" es predicativa y la ñ, "Simiente no miente" está compuesta en el sujeto por un solo elemento seguido de núcleo verbal.

La significación de los versos de la estrofa IV encuentra su correspondencia en la estrofa anterior: El significado de los versos 24 a 27 es equivalente a 20 y 21 , III. ¿Cómo se dice lo que no dice, pues "ataraxia" equivale a "silencio", en tato que posee el sema nuclear 'imperturbabilidad'. En cuanto a la respuesta a la inetrrogación, advertimos que "un grito en un cráter extinto" es una frase sinónima de "silencio" porque el adjetivo "extinto" niega el sema nuclear "expulsión violenta' de "cráter". Las citadas equivalencias localizadas en la palabra "silencio" nos dan pie para interpretar los versos 24 a 27 de la siguiente manera: "¿Cómo se dice silencio?: silencio", significación idéntica a ¿Cómo se dice lo que no dice? $(20,21)$.

Los versos 28 y 29 Lo que se dice se dice/al derecho y al 'revés se relacionan con el 23, Tal vez es bestial la vestal, donde señalamos la posibilidad de una lectura reversible.

Los versos 30 y 31 , Lamenta la mente/De menta demen- 
te/ presentan relaciones fonosemánticas que, en principio, ejemplifican nuevamente lo que ocurre en el "oído", el cual, de acuerdo al poema, es tanto "nido" como "laberinto del sonido". En estos versos las repeticiones silábicas se corresponden en número par:

\section{$\begin{array}{lll}\text { Verso } 30 & \text { Verso } 31 & \text { Versos } 30 \text { y } 31\end{array}$}

Horizontalmente

Dos veces la dos veces de

Dos veces men dos veces men
Verticalmente

Dos veces $t a$

Dos veces te

Las repeticiones integran una anafonía. ${ }^{12}$

Dentro de las equivalencias fónicas observamos que las palabras finales de 30 y 31 , "mente" y "demente" poseen los semas nucleares 'razón' y 'carencia de razón', respectivamente, hecho que los convierte en pareja antitética. La relación fonosemántica de los versos referidos enfatiza justamente la continua creación de semejanzas y oposiciones en el recorrido de la palabra.

En los últimos versos de IV, 32 y 33 , Cementerio es sementero,/Simiente no miente/ se advierte la repetición silábica siguiente:

\section{Verso $32 \quad$ Verso 33}

$\begin{array}{ll}\text { Dos veces ce }(\mathrm{se}) & \text { Dos veces } m i \\ \text { Dos veces men } & \text { Dos veces en } \\ \text { Dos veces rio }(\mathrm{ro}) & \text { Dos veces te }\end{array}$

Las sílabas que no se repiten son si y no, pero forman la antítesis "si" /"no" que podemos obtener separando la palabra "simiente" (sí dice mentiras) y la enfrentamos a "no miente". Inmersa también en los paralelismos fónicos se en-

I2 Cf. Jean Starobinski, "Los anagramas de Ferdinand de Saussure", en F. de Saussure, Fuentes manuscritas $y$ estudios criticos, Siglo XXI, México, 1977; pp. 229-230. 
cuentra la antítesis : cementerio"/"sementero" puesto que los elementos comportan los semas 'muerte' y 'vida', respectivamente. La oración equivale a decir "muerte es vida", postulándose así la disolución de los términos contrarios.

La conjunción de las equivalencias fónicas de los versos 30 a 33 evidencia que cada verso está formado por tres palabras y que en ellas ocurren las repeticiones de una parte de ellas:

\section{Versos 30 a 33}

\section{Horizontalmente}

\begin{tabular}{c|c} 
menta & menta \\
menta & mente \\
mente & mente \\
mente & mente \\
miente & miente
\end{tabular}

Esta disposición configura un hipograma ${ }^{13}$ y una pareja antitética cuyos elementos están en el primero y el último verso, siguiendo una línea vertical: "mente"_-miente". Las repeticiones de los segmentos de las palabras parecen ser el resultado de una división entre las palabras, de modo que casi se reflejan unas en otras, como si estuviesen ante un espejo; y precisamente el reflejo produce apariencias. Es evidente que "menta" - "miente" especifica que mentar, es decir, nombrar o mencionar, se opone a mentir, que contiene entre sus semas 'desdecir', con lo cual equivaldrían a "decir" / "desdecir". Pero también los semas 'falsificar' e 'inducir a error' de "mentir" conectan la referencia con "laberinto del sonido". (1S) que confunde el significado de la palabra, en última instancia, que lo vuelve ambiguo, de modo análogo a lo ocurrido a los versos a que hacemos referencia.

La estrofa $\mathrm{V}$ del poema, compuesta por cuatro versos, 34, 35,36 y 37, integra la oración o, "Laberinto del oído", lo

13 " [...] en el hipograma se trata de subrayar un nombre, una palabra, esforzándose por repetir sillabas, y dándole así una scgunda manera de ser ficticia, añadida, por asi decir, a la original de la palabra." Cf. Ibid.; p. 232. 
que dices se desdice del silencio al grito desoído". En pri. mer lugar, el verso 35, Lo que dices se desdice, es semejante a las oraciones $g y h$ de III, aunque ya aparece un sujeto determinado "tú" (dices), que es "laberinto del oído", así como también la antítesis "decir"/ "desdecir" equivalente a "decir" / "no decir" de III. "Decir"/desdecir' es equivalente a todo el juego de relaciones fonosemánticas de la estrofa IV. Finalmente, los versos 36 y 37 de V, Del silencio al grito Desoido, complemento circunstancial de la oración, reitera la formación de la pareja sinónima "silencio" = "grito desoído", así como el concepto de que la ambigüedad originada en el "laberinto del sonido" "desdice" lo "que se dice" del silencio al "silencio", esto es, que la ambigüedad reside en el poema, de acuerdo con lo expresado por Octavio Paz: "El poema es el tránsito entre uno y otro silencio - entre el querer decir y el callar que funde querer y decir." 14

La última estrofa del poema, VI, está compuesta por dos versos, 38 y 39 , que forman la oración $p$, "Inocencia y no ciencia: para hablar aprende a callar." En la oración predominan los verbos, rasgo que emparenta esta estrofa con la III; hay dos infinitivos "hablar" y "callar", semánticamente antitéticos, posición que está antecedida por la frase "inocencia y no ciencia", que actúa como una reiteración del juego de homofonías y antinomias.

La antitesis "inocencia"/"ciencia" reúne la oposición de los semas "candor' y 'simplicidad' de "inocencia", pero también añade el interpretante sémico 'fácil de engañar' (de "inocente"). "Ciencia", por su parte, contiene los semas nucleares 'saber', 'erudición' y 'conocimiento cierto'. La sentencia expresada a un tú (aprende) que sería el poeta precisa su significado si retomamos el concepto esencial postulado en el poema, de que la palabra dicha no se ajusta a un solo decir, sino que precisamente en su tránsito despliega su polisemia en el acto de la lectura, a la vez que el encuentro de sus significantes vuelve ambiguos sus significados.

Pero la sentencia final del poema ha sido expresada por

${ }^{14}$ Corriente alterna; p. 74. 
Paz en otra instancia: "Enamorado del silencio, el poeta no tiene más remedio que hablar." 15

EI análisis de "La palabra dicha" nos ha indicado que el poema se refiere a la lectura del poema; sobre ésta su autor teoriza alternando con la ejemplificación. Finalmente el sentido del acto de "decir" el poema encuentra también su definición en la teoría poética de Octavio Paz:

Entre el grito y el callar, entre el significado que es todos los significados y la ausencia de significación, el poema se levanta. ¿Qué dice ese delgado chorro de palabras? Dice que no dice nada que no hayan ya dicho el silencio y la gritería. $Y$ al decirlo, cesan el ruido y el silencio. Precaria victoria, amer nazada siempre por las palabras que no dicen nada, por el silencio que dice: nada. ${ }^{16}$

Leticia Algaba

Seminario de Poética.

Instituto de Investigaciones Filológicas. 